Conclusions Percutaneous mitral valve repair with MitraClip device is a safe and viable management option in high-risk patients with cardiogenic shock or refractory pulmonary edema and concomitant moderate-severe MR. Prospective trials are required to confirm these findings, and definitively determine the value of MitraClip in hemodynamically unstable patients.

\section{SAFETY AND EFFICACY OF TRANSCATHETER AORTIC VALVE IMPLANTATION IN NONAGENARIANS WITH SEVERE SYMPTOMATIC AORTIC STENOSIS}

PF Brennan, B Cox, A Santos, F Corvan, O Nzewi, N Johnston, A McNeice, R Jenanathan, C Owens, MS Spence, G Manoharan. Royal Victoria Hospital, Belfast, UK

\subsection{6/heartjnl-2020-ICS.29}

Background Transcatheter aortic valve implantation (TAVI) is the standard of treatment for high/prohibitive surgical risk patients with symptomatic severe aortic stenosis. Studies reported over the last 5 years have found TAVI to be noninferior to surgery with respect to intermediate and lower risk patients. In the United Kingdom the number of people living beyond 90 years age continues to rise and this had led to an increasing number of nonagenarians undergoing TAVI for symptomatic aortic stenosis. Outcomes for this group patients are scarcely reported.

Purpose We aimed to report outcomes for all nonagenarians undergoing TAVI in Northern Ireland (NI).

Methods Baseline characteristics, procedural parameters and outcomes were collected using the NICOR database, NI Electronic Care Record and our in-hospital database. We compared all 73 nonagenarians with the remaining 1048 patients that underwent TAVI in NI.

Results Nonagenarians (mean age $=91.6$ years; female sex $55 \%)$ had a lower mean EuroSCORE2 $(7.2 \%$ vs $8.5 \%$, $\mathrm{p}=0.03$ ) than the younger group (mean age $=81$ years; female sex $53 \%)$. COPD $(4.1 \%$ vs. $20.3 \%, \mathrm{p}<0.005)$, diabetes mellitus $(11 \%$ vs. $22.3 \%$, p 0.02$)$, prior cardiac surgery $(6.8 \%$ vs. $19.8 \%, \mathrm{p} 0.01)$ were all significantly lower in the nonagenarian patients. Nonagenarians had significantly more progressive renal dysfunction (mean creatinine clearance $36.4 \mathrm{ml} / \mathrm{min}$ vs. $48.3 \mathrm{ml} / \mathrm{min}, \mathrm{p}<0.005)$.

Left ventricular dysfunction $(\mathrm{LVEF}<50 \%)$ was less prevalent in the nonagenarians ( $14 \%$ vs $28 \%$, p 0.01). TAVI was performed under local anaesthetic (LA) in $97 \%$ of nonagenarians, compared to $95.5 \%$ younger patients, for which 93\% vascular access was percutaneous femoral (pTF) (nonnonagenarians LA 98\%, pTF 92.2\%). Surgical femoral and axillary/subclavian made up the remainder of the access for nonagenarians.

Procedural complications for nonagenarians vs the remaining group included stroke $(4.3 \%$ vs $2.82 \%$, p 0.22), tamponade $(1.4 \%$ vs $1 \%, \mathrm{p} 0.61)$ and major vascular injury $(1.4 \%$ vs $3.4 \%$, p 0.62 ). $30 \mathrm{~d}$ new pacemaker implantation was similar between the nonagenarian and younger patients (12 vs. $13 \%$, p 0.78). Peri-procedural mortality trended higher in nonagenarians at $4 \%$ vs $2 \%$, p 0.20 but, overall, $30 \mathrm{~d}(4 \%$ vs. $3 \%, \mathrm{p} 0.47)$ and $1 \mathrm{y}(16 \%$ vs. $10 \%, \mathrm{p} 0.10)$ mortality did not significantly differ when compared with the younger patient group. The median survival estimate (figure 1) for nonagenarians was 4.9y (IQR 2.3-7.4y) compared to 6.1y (IQR 3.1$9.1 y$ ) for the younger patient group ( $p$ 0.10). Readmission for

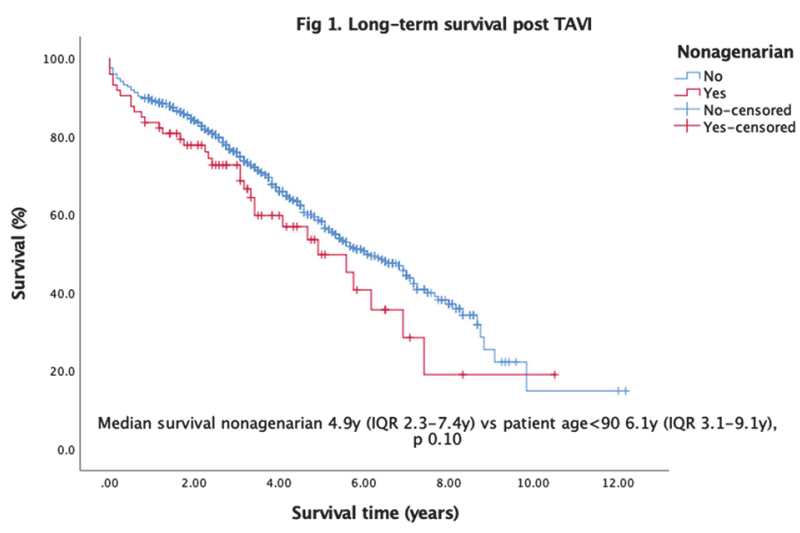

Abstract 29 Figure 1 The median survival estimate

heart failure within 1-year was non-significantly lower for nonagenarians (6\% vs $10 \%$, p 0.23).

Conclusions In selected nonagenarians we severe symptomatic aortic stenosis, TAVI is both safe and efficacious achieving acceptable short- and long-term outcomes that are comparable with younger patients undergoing TAVI.

\section{SYNTAX SCORE- A SIMPLE WAY TO STRATIFY COMPLEX CORONARY DISEASE?}

J Mailey, C Murphy. Royal Victoria Hospital, Belfast, UK

\subsection{6/heartjnl-2020-ICS.30}

Background The Syntax Score (SS) is an angiographic risk stratification tool to grade coronary disease complexity and aid revascularisation strategy decision making. Calculated scores are divided into tertiles $(0-22,23-32,>32)$ and guidelines advocate that decisions should be based on this tertile (1). Syntax Score calculation is dependent on the subjective interpretation of coronary angiograms by Interventional Cardiologists (ICs) and is therefore prone to variability. Our institution consists of 13 ICs performing on average >5000 coronary procedures per year. We sought to compare syntax scoring amongst ICs.

Methods 10 patients with multi-vessel coronary artery disease who had previously been discussed by a Multi-Disciplinary team (MDT) were retrospectively identified. Eight ICs were asked to interpret the coronary angiograms and calculate a SS for each patient. Scoring was performed independently, with no knowledge of the score that had been used at the MDT discussion. Interobserver variability between raters for the raw syntax score was assessed using intraclass correlation coefficient. Interobserver variability between raters with respect to tertile was assessed using Fleiss kappa.

Results All 8 ICs completed scores for each patient. Mean calculated SS was $30.2( \pm 6.7$ SD). $21.3 \%$ of scores were $1 \mathrm{st}$ tertile (0-22), $45.0 \%$ were 2 nd tertile (23-32) and $33.7 \%$ were 3rd tertile $(>33)$. Mean range of calculated SS between raters was 18.5 (range 9.5 - 29). 40.0\% of scores were divided across all 3 tertiles with the remaining $60.0 \%$ divided across 2 tertiles. Intraclass correlation coefficient of raw SS was 0.55 suggesting only fair agreement. The interobserver strength of agreement with regard to tertiles was also only fair (Fleiss Kappa $=0.25$; 95\% CI [0.07, 0.43]). The interobserver strength of agreement improved to moderate when comparing tertile 1 vs tertile 2 or 3 together (Fleiss 
Kappa $=0.47 ; 95 \%$ CI $[0.17,0.77])$. Only $63.1 \%$ of IC tertile scores agreed with that quoted at the initial MDT. This improved to $82.3 \%$ when comparing to tertile 1 vs tertile 2 or 3 .

Conclusion Overall we demonstrated only fair to modest repeatability in SS calculation amongst individual ICs. This is similar to findings in other studies, though it is known improvements are possible with intensive training (2). We advise that significant variability in SS calculations should be considered within all institutions unless validated training systems are in place. In the absence of these systems we suggest the weight of importance attributed to quoted SS in MDT decision making should be guarded.

\section{OUTCOMES OF ACCESS SITE IN PATIENTS WITH STEMI}

D Ranganathan, R Howley, P O'Connor, P Kearney. Cork University Hospital, Cork, Ireland

\subsection{6/heartjn|-2020-ICS.31}

Introduction Current ESC Guidelines (2017) recommends radial access over femoral access (Class 1A ) during primary PCI for STEMI. However, radial access can be technically more challenging, particularly in older patients, smaller patients and females in whom the radial artery may be small and brachiaand brachocephalic tortuously is frequently encountered. This may delay re-perfusion, and increase radiation exposure.

Aim We aimed to analyse real-world clinical data on the choice of access site with success of intervention, complications rate and radiation exposure in patients presenting with STEMI.

Methods We retrospectively evaluated 227 patients presenting to Cork University Hospital between January 2019 to December 2019 with a ST-elevation myocardial infarction. 58 patients were excluded due to lack of sufficient data and the remaining 169 patients were compared based on their access site.

Observed variables included success of intervention, allcause mortality, complication rate and radiation exposure. Outcomes of patients operated via the radial approach versus the femoral approach was compared using Chi-Square. We used SPSS for all statistical analysis and p-values of $<0.05$ was considered to be statistically significant.

Results The demographics of the 169 patients analysed are described (table 1). 120 patients $(71 \%)$ had a radial artery for access of which 7 patients transitioned to an alternative access site (5 patients to right femoral artery and 2 patients to left radial artery).

Of the 49 patients in femoral access site, 6 patients had no palpable right radial pulse/Barbeau's test negative, 5 were anticipated to require intra-aortic balloon pump/temporary wire insertion and 1 had flexure contracture of right arm from previous stroke. Of 5 patients in the left radial access, 2 had previous $\mathrm{CABG}, 2$ had no palpable right radial pulse and 1 had AV fistula involving right forearm.

Fourteen patients were thrombolysed at another centre, 1 brought for immediate PPCI despite successful lysis and 13 required rescue PCI. There were 3 unsuccessful cases between the 2 groups (p-valve 0.283 ). The patients with graft study in each group had successful interventions regardless of access site. When comparing radiation exposure, there was no significant difference between radial and femoral
Abstract 31 Table 1 Baseline characteristics, procedural data, and outcomes

\begin{tabular}{|c|c|c|}
\hline & $\begin{array}{c}\text { Radial }(n=120)-5 \\
\text { changeover to femoral } \\
\text { access }\end{array}$ & Femoral $(n=49)$ \\
\hline Age & 61.8 & 65.3 \\
\hline Males & 100 & 39 \\
\hline BMI & 28.9 & 27.3 \\
\hline HTN & 46 & 24 \\
\hline Dyslipidaemia & 37 & 19 \\
\hline DM & 23 & 10 \\
\hline Current smoker & 40 & 16 \\
\hline Thrombolysis & $\begin{array}{c}11 \\
\text { Failed: } 10\end{array}$ & $\stackrel{3}{\text { Failed }: 3}$ \\
\hline Left sided access & 5 & None \\
\hline Graft studies & 2 & 3 \\
\hline Culprit vessel & $\begin{array}{lc}- & \text { RCA: } 47 \\
- & \text { LMS: } 2 \\
- & \text { LAD: } 49 \\
- & \text { LCX/RI: } 18 \\
- & \text { Graft: } 1 \\
- & \text { No intervention: } 3\end{array}$ & $\begin{array}{lc}- & \text { RCA: } 27 \\
- & \text { LMS: } 1 \\
- & \text { LAD: } 18 \\
- & \text { LCX: } 2 \\
- & \text { Graft: } 1 \\
- & \text { No intervention: } 0\end{array}$ \\
\hline $\begin{array}{l}\text { Rate of unsuccessful } \\
\text { intervention }\end{array}$ & 3 & 3 \\
\hline Mean fluoroscope time & 12.42 & 12.01 \\
\hline $\begin{array}{l}\text { Mean Dose area product } \\
\text { (mGycm2) }\end{array}$ & 55,432 & 45,351 \\
\hline $\begin{array}{l}\text { Average change in creatinine } \\
\text { pre/1-day post angiogram }\end{array}$ & $14 \%$ increase & $23 \%$ increase \\
\hline Complications & $\begin{array}{lc}- & 1 \text { Stroke } \\
- & 1 \text { Acute kidney injury }\end{array}$ & $\begin{array}{l}\text { - } 1 \text { Retro-peritoneal bleed } \\
\text { - } \quad 3 \text { Acute kidney injury }\end{array}$ \\
\hline Mortality & $4(3.33 \%)$ & $6(12 \%)$ \\
\hline
\end{tabular}

access on fluoroscopy timing and dose area product ( $\mathrm{p}$-value $0.2425)$. There was a higher average creatinine rise associated with femoral access, possibly a consequence of higher contrast volume. In regard to complications, there was higher mortality risk associated with femoral access (p-value 0.038) and complication rates ( $p$-value 0.048 ) and significant overall adverse outcomes (p-value 0.005986) compared to radial access.

Conclusion Complication rates were very low, but our study findings support previous studies, such as MATRIX, RIFLESTEACS and RIVAL, with regard to lower vascular complications associated with radial access. The study revealed similar radiation exposure levels between the 2 access sites.

\section{General poster session}

\section{IMAGE NOT IMAGINE. POCUS AND DISCHARGE DECISION MAKING IN HOSPITALIZED PATIENTS WITH HEART FAILURE}

L O'Toole, P Sharma, M Malik, S Rummun, S Fleming. Midlands Regional Hospital, Portlaoise, Co. Laois, Ireland

\subsection{6/heartjnl-2020-ICS.32}

Introduction Among patients hospitalized with heart failure (HF), residual congestion (RC) at discharge is common and predicts rehospitalization and death at 6 months. Clinical assessment of congestion is challenging particularly for hospitalized patients as they near discharge. Point of Care Ultrasound (POCUS) is an emerging modality offering advantages of accessibility and convenience over traditional imaging 